\section{European Association for the Study of the Liver}

The third meeting of the European Association for the Study of the Liver was held in Modena from September 19 to 22, 1968, under the Presidency of Professor Mario Coppo (Modena) and with the active participation of members from 15 European countries. It is a pleasure to record that Czechosl vakia was represented by Professor Hoenig (Prague) and two of his fellow countrymen. Sessional chairmen included Professors S. Sherlock (London), A. Martini (Marburg), J. Caroli (Paris), S. Bengmark (Göteborg), and H. Thaler (Vienna).

\section{PATHOLOGY}

A new classification for chronic hepatitis was recommended by a committee of pathologists representing most European countries. Their proposal is a subdivision into chronic persistent and aggressive types of hepatitis. Chronic persistent hepatitis comprises chronic inflammatory infiltration, mostly portal, with preserved lobular architecture and little or no fibrosis. Features of acute hepatitis may be superimposed. Chronic aggressive hepatitis involves portal tracts and spreads into the parenchyma, with piecemeal necrosis and formation of intralobular septa. Architecture is disturbed but there is no nodular regeneration. Activity, as shown by piecemeal necrosis and inflammation, can be subdivided still further into moderate and severe.

\section{XANTHINE OXIDASE ENZYME}

DR DE GROOTE and his co-workers (Louvain) reported elevated serum xanthine oxidase activity in acute liver disease, correlating with transaminase values.

Allopurinol (hydroxypyrazolo-pyrimidine) is a potent inhibitor of xanthine oxidase, so its effect in blocking various biochemical pathways has aroused considerable interest. DRS VENTURA and CALANDRA (Modena) have observed the effect of allopurinol on purine metabolism in normal subjects and in patients with alcoholic liver disease. Ethanol was administered intravenously to these subjects over a four-hour period as $500 \mathrm{ml}$ of a $13.5 \%$ solution in isotonic saline. In alcoholic patients a marked increase in the serum uric acid level occurred, whilst its renal excretion was reduced without significant changes in glomerular filtration rate. Similar effects but to a lesser extent were noted in normal subjects. The effect of alcohol on renal uric acid excretion was related to a rise of blood lactate during hepatic metabolism of ethanol by alcohol-dehydrogenase and a consequent fall in the pyruvate level. After treatment with allopurinol over five days, alcohol infusion did not produce any significant change in serum uric acid, lactate and pyruvate levels in either normal or alcoholic subjects. After five days, treatment with sulfinpyrazone, a powerful uricosuric agent, also blocked hyperuricaemia due to alcohol, but raised serum lactate and decreased pyruvate levels were noted. DRS STROHMEYER, CHEARANAI, and GOEBELL (Marburg/Lahn) analysed the effect of allopurinol on iron metabolism through the ferritin-xanthine oxidase system. They showed that allopurinol affects xanthine oxidase in the liver but not in the intestinal mucosa.

\section{IMMUNOLOGY}

DR R. A. FOX and his colleagues (London) have observed in primary biliary cirrhosis an altered immunological state as reflected by tissue granulomas and by depression of delayed-type hypersensitivity. Granulomatous reactions were noted in 27 of $63(43 \%)$ patients, principally in the liver, but in five patients they were found in lymph nodes, omentum, or lung. Sarcoidosis was excluded by the absence of a compatible clinical picture in lungs, eyes, or skin, and by negative Kveim tests in eight patients tested. The mitochondrial immunofluorescent test was positive in 17 of 18 patients with granulomas. Depression of delayed-type hypersensitivity was manifest by an increased incidence of negative skin tests using tuberculin, candida, and dinitrochlorobenzene and was correlated with a similar lack of response of patients' lymphocytes to tuberculin or to phytohaemagglutinin by the in vitro lymphocyte transformation test.

\section{BILIRUBIN METABOLISM}

DRS BLACK and BILLING (London) have adapted a new method for the assay of glucuronyl transferase so that it can be applied to small amounts of liver tissue. Hepatic bilirubin UDP glucuronyl transferase activity, which appears to be the limiting factor in the biliary excretion of bilirubin as bilirubin glucuronide, is significantly reduced in Gilbert's congenital hyperbilirubinaemia. Disorders of hepatic parenchyma are not associated with reduced enzyme activity but cholestasis causes augmentation of this activity.

DRS BRATLID and FOG (Oslo) discussed some factors affecting kernicterus, which develops when inefficient or defective hepatic conjugation by glucuronyl transferase allows unconjugated bilirubin to rise to a level of over $20 \mathrm{mg}$ per $100 \mathrm{ml}$ plasma. Plasma albumin is soon saturated and is no longer capable of retaining, within the vascular system, this pigment which consequently drains out into the tissues, including the brain. This seems to happen when the molecular ratio of bilirubin:albumin is $2: 1$.

DRS KUCEROVA and HOENIG (Prague) have found that conjugated bilirubin is bound less firmly to albumin than unconjugated bilirubin. Both bilirubins were more or less displaced from albumin by sodium oleate, sodium glycocholate, sodium dehydrocholate, and bromsulphthalein. Unexpectedly, sodium oleate liberated unconjugated bilirubin readily, perhaps because of a greater number of common binding sites shared by sodium oleate and unconjugated bilirubin.

\section{HAEMODYNAMICS}

DR J. P. BENhAMON and his colleagues (Paris, Clichy) have measured the mean transit time of ${ }^{51} \mathrm{Cr}$-labelled 
erythrocytes and of ${ }^{125} \mathrm{I}$-labelled albumin in the hepatic artery and portal vein of the normal dog's liver. The sinusoidal transit time is longer in the hepatic artery than in the portal vein and the reverse holds for the nonsinusoidal time. The difference suggests that arterial and portal venous blood flows are largely independent of each other within the liver. The experiments also indicate that circulation is slower in sinusoids perfused by the hepatic artery rather than in those perfused by the portal vein. The hepatic artery transit time is very long in patients with hepatic cirrhosis.

DRS SOVAK and KARDEL (Copenhagen) demonstrated an elegant new biplane cinesangiography technique for visualizing the portal venous tree and collaterals and for measuring portal flow in dogs, using a new particulate non-embolizing medium (Mygind, 1966) and small droplets of Lipiodol ultra-fluid. The flow was calculated from the mean velocity of the particles and the mean cross-sectional area of the portal vein. DR W. A. FUCHS and his co-workers (Bern) defined hepatic arterial steal as a redistribution of perfusion in cirrhotic patients in favour of arterial inflow. There is retrograde filling of the pancreatic arcade, gastroduodenal artery, and interhepatic arteries. This compensatory mechanism increases hepatic blood flow when coeliac axis inflow is reduced and as such it benefits the liver, but it may do so at the expense of the circulation to the pancreas and small intestine. Hepatic artery steal is noted in those cirrhotics with splenomegaly, raised wedged hepatic vein pressure, and prolonged bromsulphthalein retention.

DRS N. GITLIN and co-workers (London) have studied the splenic blood flow and resistance in patients with well-compensated cirrhosis before and after portacaval anastomosis. This operation led to decreased size of the spleen, decreased splenic resistance, and increased splenic blood flow. A somewhat similar change was noted by DR B. PORRO and colleagues (Milan) in cerebral blood flow after portacaval shunt operations; there was a significantly increased cerebral blood flow and decreased vascular resistance.

DRS L. M. BLENDIS and co-workers (London) have found that in patients with normal or elevated red cell mass there is also an increase in plasma volume resulting in a total blood volume greatly in excess of the predicted value. There was a close correlation between plasma volume and spleen size in patients with blood dyscrasias but not in cirrhotics. Medical treatment or splenectomy in the blood dyscrasia group resulted in little fall in the plasma volume though in the following nine months there was a slow return to normal.

\section{HORMONES}

DRS GERDES and STROHMEYER (Marburg) have demonstrated a qualitative and quantitative change in metabolic transformation of cortisol in hepatic cirrhosis, supporting the concept that progressive deterioration in hepatic degradation of cortisol upsets the feedback mechanism of hepatic regulation of pituitary ACTH secretion leading to pituitary suppression, reduced adrenocortical cortisol production, and a diminished diurnal variation of free cortisol and its metabolites.

\section{Comment}

Moore (Gastroenterology, 1968, 54, 501-507) showed that if a glass electrode was calibrated only at $p \mathrm{H} 7$ measurements in the range $p H 1$ to 3 were likely to show large errors. Was this factor taken into account in the study by Lennard-Jones and his colleagues (Gut, 1968, 9, 177-182) on the effect of foods on acidity of gastric contents? If not, how might such errors affect the results?

\section{Veterans Administration Center, LOS Angeles, California}

As described in the original paper (Gut, 6, 113-177) the $p \mathrm{H}$ meter was calibrated at $p \mathrm{H} 4.0$ as well as at $p \mathrm{H} 7 \cdot 0$. Moore's experiments demonstrate the validity of calculating hydrogen ion concentrations from measured $p \mathrm{H}$ values in hydrochloric acid solutions and gastric juice of known ionic strength obtained from fasting subjects. In gastric juice containing food the situation is more complex, as the effect of protein on the liquid junction potential is not known and the ionic strength varies. For these reasons no attempt has been made to derive hydrogen ion concentrations from the $\mathrm{pH}$ measurements made on gastric contents after food. The absolute accuracy of the results may have been affected by the fact that the $p \mathrm{H}$ meter was not calibrated in the range $p \mathrm{H} 1$ to $p \mathrm{H} \mathrm{3.} \mathrm{Moore} \mathrm{has} \mathrm{shown} \mathrm{that} \mathrm{calibration} \mathrm{with}$ commercial buffers produces a constant bias and in these experiments on the effect of food on the acidity of the gastric contents any such bias is likely to have affected results with different diets in the same way.

J. E. LENNARD-JONES University College Hospital, London

European Association for the Study of the Liverconcluded

After parenteral administration of testosterone to the male cirrhotic, DR P. GREGERSEN (Copenhagen) noted a reduced recovery of urinary 17 -ketosteroids, suggesting abnormal androgen metabolism rather than primary testicular insufficiency as a cause of feminization. DRS DELLA CASA and colleagues (Modena) have investigated oestrogen metabolism in cirrhotics. The basal excretion of oestrogen was significantly higher in cirrhotics than in healthy subjects, but there were no significant differences between cirrhotics with or without feminization. ACTH stimulation and dexamethasone suppression did not influence appreciably the excretion of oestrogen in normal subjects but resulted in significant elevation of urinary oestrogen excretion in cirrhotics with signs of hyperoestrogenism. Cirrhotics without feminization behaved intermedially between normals and feminized cirrhotics.

D. GERAINT JAMES 